\title{
Wigner crystallization in semiconductor quantum wires
}

\author{
B. Tanatar \\ Department of Physics, Bilkent University, Bilkent, 06533 Ankara, Turkey \\ I. Al-Hayek and M. Tomak \\ Department of Physics, Middle East Technical University, 06531 Ankara, Turkey \\ (Received 29 April 1998; revised manuscript received 29 June 1998)
}

\begin{abstract}
We study the Wigner crystallization in semiconductor quantum wires within the density-functional approach. As the density of electrons in quasi-one-dimensional structures is lowered, we find that the system favors the crystalline phase as envisioned by Wigner. The dependence of the critical density on the lateral width of the quantum wire is also investigated. In a structure consisting of two parallel quantum wires, the Wigner transition to the solid phase is enhanced similarly to the bilayer systems. [S0163-1829(98)01039-X]
\end{abstract}

One-dimensional (1D) electron-gas models are of great interest because of theoretical and technological implications. The motion of electrons confined to move freely only in one spatial dimension gives rise to a variety of interesting phenomena. The prospect of observing non-Fermi-liquid (i.e., Luttinger liquid) characteristics has been a large impetus on part of the current research in 1D electron systems. Various transport and optical properties are important topics of investigation for our understanding and potential utilization in mesoscopic devices. ${ }^{1}$ Because of the reduced scattering and energy-loss rates, quasi-one-dimensional (Q1D) electron systems as realized in semiconductor structures are also under intense study. ${ }^{2}$ Many-body effects in quantum wires have recently gained importance as the fabrication techniques continue to improve.

In this work we investigate the possibility of fluid to solid phase transition in a Q1D electron system at low densities. The so-called Wigner transition ${ }^{3,4}$ occurs at zero temperature, ${ }^{5}$ and at a critical density when the Coulomb energy is much larger than the kinetic energy. This manybody phenomenon originally envisaged for a threedimensional system has been observed in a 2D layer of electrons on the surface of liquid helium. ${ }^{6}$ The realization of a Wigner crystal in the quantum regime is largely hindered by disorder-induced localization effects. Although a number of researchers have investigated the properties of onedimensional electrons in the crystalline phase, ${ }^{7-10}$ no estimate was given for the transition density at zero temperature. Our chief aim in this work is to provide an estimate on the 1D electron density at which a Wigner solid forms within a simple jellium model and available exchange-correlation energies. We find that semiconductor-based heterostructures out of which Q1D electronic systems can be formed promises to be a likely candidate to observe the quantum freezing transition of a $1 \mathrm{D}$ electron system. It is interesting to note that the existence of excitonic crystals in Q1D semiconductor quantum wires were predicted by Ivanov and Haug ${ }^{11}$ for densities $1<r_{s} \leqslant 5.5$. Our calculations are in qualitative agreement with this result for the comparable structures.

The density-functional theory of freezing of quantum liquids has been quite successful in predicting the critical values of thermodynamic parameters at the liquid-solid transition and in describing the nature of the transition. The pioneering work of Ramakrishnan and Yussouff, ${ }^{12}$ as devel- oped by Senatore and Pastore, ${ }^{13}$ with application to quantum systems sets the theoretical framework for the present study. In the density-functional theory of freezing, ${ }^{14}$ first a suitable energy functional of the charge density is set up. Then the homogeneous and inhomogeneous phases (treated on an equal footing) are identified through some energyminimization procedure. Below, we briefly outline the method we use, and present our results for the Wigner crystallization in single- and double-wire electron systems.

The ground state energy $E_{0}$ of a many-electron system can be written as a functional of the electron density $\rho(\vec{r})$ as

$$
\begin{aligned}
E_{0}[\rho]= & \int d \vec{r}\left\{t[\rho(\vec{r})]+u[\rho(\vec{r})]+E_{x}[\rho(\vec{r})]+E_{c}[\rho(\vec{r})]\right\} \\
& +\int d \vec{r} V_{e x t}[\rho(\vec{r})]
\end{aligned}
$$

where $t$ is the kinetic energy, $u$ is the Coulomb interaction potential, $E_{x}$ and $E_{c}$ are the exchange and the correlation energies, respectively. They are all functionals of the density $\rho$. $V_{\text {ext }}$ is the externally applied potential, which is taken to be zero in this work. We employ the above energy functional to a Q1D electron gas, modeled by a quantum wire with infinite length along the $z$ axis. The explicit form of the functionals to be used in Eq. (1) depends on the nature of the problem at hand. As remarked by Choudhury and Ghosh, ${ }^{15}$ the success of the method relies on various cancellations. We choose to follow the previous examples ${ }^{15-17}$ which yield reasonable estimates for Wigner crystallization in higher dimensions.

The kinetic energy functional within the Thomas-FermiWeizsäcker approximation in one dimension can be described in atomic units (a.u.) as

$$
t[\rho]=C_{k} \rho(z)^{3}+\frac{1}{8} \frac{\vec{\nabla} \rho(z) \vec{\nabla} \rho(z)}{\rho(z)},
$$

where $C_{k}=\pi^{2} / 24$, and the potential energy by

$$
u(z)=\frac{1}{2} \int d z^{\prime} \frac{\left[\rho\left(z^{\prime}\right)-\rho_{0}\right]\left[\rho\left(z^{\prime}\right)-\rho_{0}\right]}{\left[b^{2}+\left(z-z^{\prime}\right)^{2}\right]^{1 / 2}},
$$

where $\rho_{0}$ is the homogeneous electron density, and $b$ is the model-dependent quantum wire width. The above form of 
the kinetic energy is supposed to work well for highly inhomogeneous systems. In this work, we use the cylindrical quantum wire model developed by Gold and Ghazali $;{ }^{18}$ thus the electrons are confined to have free motion in the axial direction of the cylinder with radius $R=b$. We also assume that only the lowest subband of the $1 \mathrm{D}$ electron gas is populated, which becomes valid for $r_{s} \geq b / 4 a_{B}^{*}$.

For the exchange and correlation energies we employ a Padé approximation to obtain an analytical fit to the groundstate energy results obtained by Calmels and Gold ${ }^{19}$ for a Q1D electron gas. We obtain (in a.u.)

$$
E_{x}[\rho]=-\frac{1}{2} a_{0} \int d z \rho(z) \frac{1+a_{1} r_{s}+a_{2} r_{s}^{2}}{1+a_{3} r_{s}+a_{4} r_{s}^{2}+a_{5} r_{s}^{3}}
$$

and

$$
E_{c}[\rho]=-\frac{1}{2} c_{0} \int d z \rho(z) \frac{-1+c_{1} r_{s}+c_{2} r_{s}^{2}}{1+c_{3} r_{s}+c_{4} r_{s}^{2}+c_{5} r_{s}^{3}},
$$

in which $\left\{a_{i}\right\}$ and $\left\{c_{i}\right\}$ are parameters that depend on the wire radius (or lateral width). The Wigner-Seitz radius $r_{s}$ for a one-dimensional system is defined as $r_{s}=1 /\left(2 \rho_{0} a_{B}^{*}\right)$, where $a_{B}^{*}$ is the effective Bohr radius. In this work the valley degeneracy $g_{\nu}$ is taken to be unity, where the electron density $\rho_{0}$ dependence on $g_{\nu}$ is given by the relation $\rho_{0}$ $=2 g_{\nu} k_{F} / \pi$, and $k_{F}$ is the Fermi wave number in one dimension. The choice of the above parametrization is merely a matter of convenience, and we have checked that the numerical data of Calmels and Gold ${ }^{19}$ are faithfully represented, even though the Padé form of the exchange-correlation energies do not contain the suggested logarithmic terms.

To describe the inhomogeneous density distribution, we consider a small modulation of the density around $\rho_{0}$ described by

$$
\rho(z)=\rho_{0}[1+\lambda \cos (q z)],
$$

where $\lambda$ is a small parameter $(\lambda \ll 1)$, and $q$ represents a wave vector for density modulation in one dimension. Such an ansatz to the inhomogeneous density provided ${ }^{15}$ a good estimate for the Wigner transition in two dimensions. We assume that the electrons will be equidistant on a straight line in the solid phase which gives $q=2 \pi / r_{s}$. By making use of Eqs. (1)-(5), one obtains the energy difference between the solid (inhomogeneous) and liquid (homogeneous) phases $\left(\Delta E=E_{s}-E_{l}\right)$; the energy difference per particle ( $\Delta \varepsilon=\Delta E / \rho_{0} \int d z$ ), to second order in $\lambda$, in a.u., is

$$
\begin{aligned}
\frac{\Delta \varepsilon}{\lambda^{2}}= & C_{k} \rho_{0}^{2}+\frac{1}{16} q^{2}+\frac{1}{2} \rho_{0} K_{0}(q b)-\frac{1}{4}\left(\frac{a_{0}}{\rho_{0}}\right) \\
& \times\left[\frac{C D-B E-A(F-E)}{D^{2}}\right]-\frac{1}{4}\left(\frac{c_{0}}{\rho_{0}}\right) \\
& \times\left[\frac{I J-H K-G(L-K)}{J^{2}}\right],
\end{aligned}
$$

where $K_{0}(x)$ is the modified Bessel function of zeroth order. The first set of coefficients $(A-G)$ are explicitly given by

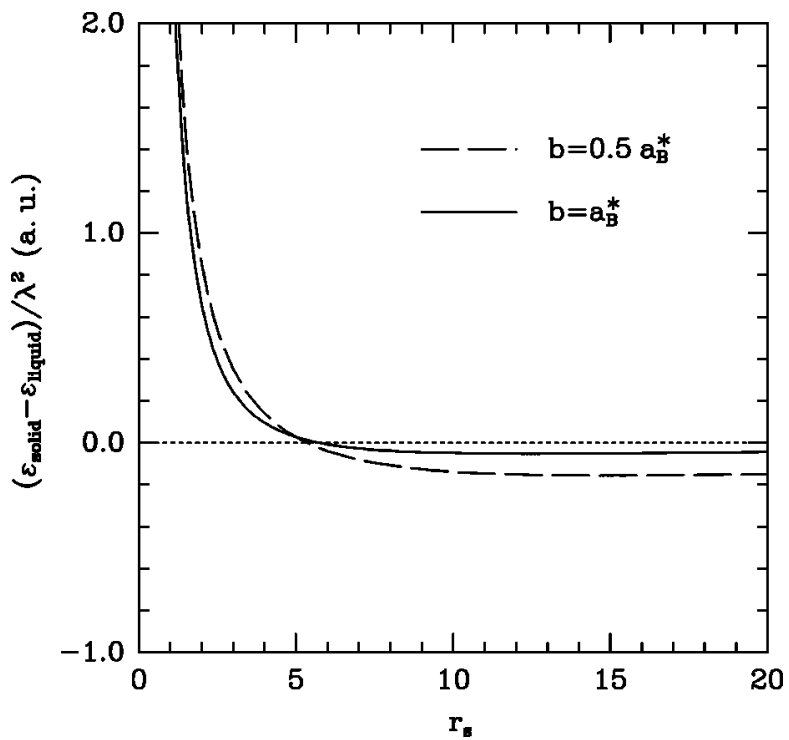

FIG. 1. The difference in the average energy per particle $\left(\varepsilon_{\text {solid }}-\varepsilon_{\text {liquid }}\right) / \lambda^{2}$ (in a.u.) as a function of $r_{s}$ for a single quantum wire of radius $b=0.5 a_{B}^{*}$ (dashed line) and $b=a_{B}^{*}$ (solid line).

$$
\begin{gathered}
A=\rho_{0}\left(1+\frac{1}{2} a_{1} \rho_{0}+\frac{1}{4} a_{2} \rho_{0}^{2}\right), \\
B=\rho_{0}\left(1+a_{1} \rho_{0}+\frac{3}{4} a_{2} \rho_{0}^{2}\right), \\
C=\frac{1}{2} \rho_{0}\left(a_{1} \rho_{0}+\frac{3}{2} a_{2} \rho_{0}^{2}\right), \\
D=1+\frac{1}{2} a_{3} \rho_{0}+\frac{1}{4} a_{4} \rho_{0}^{2}+\frac{1}{8} a_{5} \rho^{3}, \\
E=\frac{1}{2} \rho_{0}\left(a_{3}+a_{4} \rho_{0}+\frac{3}{4} a_{5} \rho_{0}^{2}\right), \\
F=\frac{1}{4} \rho_{0}\left(a_{4} \rho_{0}+\frac{3}{2} a_{5} \rho_{0}^{2}\right), \\
G=\rho_{0}\left(-1+\frac{1}{2} c_{1} \rho_{0}+\frac{1}{4} c_{2} \rho_{o}^{2}\right) .
\end{gathered}
$$

The second set of coefficients $(H-L)$ can be obtained from the first set by interchanging $\left\{a_{i}\right\}$ and $\left\{c_{i}\right\}$. It has been commented $^{20}$ that the above procedure of using a modulated density results in a rather poor density profile, although a reasonable estimate of the fluid-solid transition is obtained. Another popular form for the density distribution is a Gaussian with variational parameters, ${ }^{15,17}$ which predicts the transition density rather well and gives reasonable density profiles in 2D electron systems. Since our primary aim here is to obtain an estimate for the freezing densities in 1D structures, we have not attempted a Gaussian ansatz for $\rho(z)$.

Plotting Eq. (7) as a function of the density parameter $r_{s}$ in Fig. 1, the freezing point is determined to occur at $r_{s c}$ $=5.355$ for wire radius $0.5 a_{B}^{*}$, and at $r_{s c}=5.717$ for wire radius $a_{B}^{*}$. These values are in good agreement with the excitonic Wigner crystal calculations of Ivanov and Haug. ${ }^{11}$ We were not able to obtain a transition to the ordered phase for quantum wires of radius $b \gtrsim 2 a_{B}^{*}$. This, however, may be partly due to the approximate nature of the correlation energy. Currently produced quantum wires have densities of $r_{s} \approx 1$, but it is conceivable that advances in the fabrication techniques will lead to the observation of Wigner crystallization in such structures. To obtain a qualitative picture of the quantum wire width dependence of the phase transition, 
we calculate $\langle V\rangle /\langle K\rangle=\Gamma_{0}$ in the fluid phase, where $\Gamma_{0}$ is some constant. ${ }^{21}$ Approximating the average potential energy of a quantum wire by $\langle V\rangle=e^{2} /\left(r_{s}^{2} a_{B}^{* 2}+b^{2}\right)^{1 / 2}$ and the kinetic energy (at zero temperature) by $\langle K\rangle=\pi^{2} n^{2} / 24 m$, at the crystallization we derive the relation

$$
\frac{1}{r_{s c}^{2}}=\frac{1}{2\left(b / a_{B}^{*}\right)^{2}}\left\{\left[1+\left(\frac{24}{\pi^{2}}\right)^{2} \frac{64\left(b / a_{B}^{*}\right)^{2}}{\Gamma_{0}^{2}}\right]^{1 / 2}-1\right\},
$$

which shows the dependence of $r_{s c}$ on the quantum wire width $b$. Our numerical calculations yield $\Gamma_{0} \approx 50$ for Q1D electron systems. Figure 2 shows $r_{s c}$ as a function of $b$ based on Eq. (9) for various $\Gamma_{0}$ values (also see Table I. Within this simple approximating procedure, crystallization for all values of $b$ is predicted. Even though there is an ambiguity in choosing $\Gamma_{0}$, making the determination of $r_{s c}$ less reliable, we believe the qualitative and quantitative picture described by this approach should be useful. The above densityfunctional theory results for the critical density at which the Wigner transition takes place are limited by the accuracy of the correlation energies used. In Q1D electron gas systems, there is no accurate quantum Monte Carlo (QMC) groundstate energy calculation. The exchange-correlation energies we use are based on the self-consistent-field method, ${ }^{19}$ which may underestimate the exact values.

The prohibitively large crystallization densities in 2D electron gas led to the suggestion that a double-layer system may enhance the possibility. ${ }^{22}$ In fact, Wigner crystallization in double layers under high magnetic field has been observed. $^{23}$ At zero magnetic field, density-functional theory-based ${ }^{17}$ calculations and other approaches ${ }^{24}$ have confirmed this view. We extend our calculations to obtain an estimate for the critical density at which a Wigner crystallization occurs in a double-wire system. We consider a double-wire system consisting of two parallel quantum wires of equal width (radius), separated by a distance $d$. The ground-state energy functional for the system $E\left[\rho^{(1)}, \rho^{(2)}\right]$

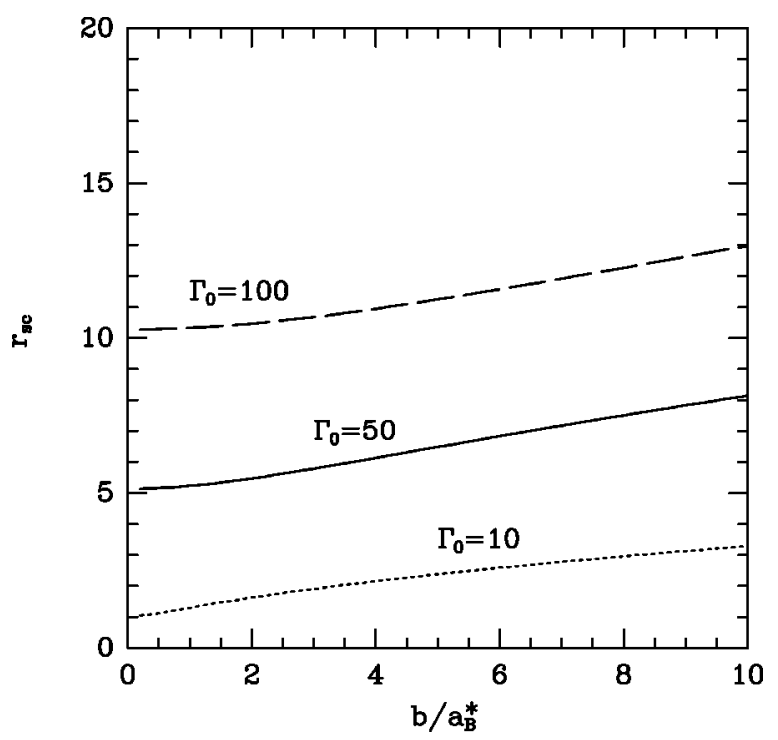

FIG. 2. The critical density parameter $r_{s c}$, at which Wigner crystallization occurs in a single wire as a function of the wire radius $b$ for $\Gamma_{0}=10$ (dotted line), $\Gamma_{0}=50$ (dashed line), and $\Gamma_{0}$ $=100($ solid line $)$.
TABLE I. The exchange and correlation energy coefficients for quantum wire radius $\mathrm{b}=0.5 a_{B}^{*}$ and $a_{B}^{*}$.

\begin{tabular}{ccccccc}
\hline \hline$b / a_{B}^{*}$ & $a_{0}$ & $a_{1}$ & $a_{2}$ & $a_{3}$ & $a_{4}$ & $a_{5}$ \\
\hline 0.5 & 4.80426 & 22.9817 & 9.611592 & 29.2405 & 84.5466 & 15.1783 \\
1.0 & 2.4023 & 13.4298 & 3.5108 & 16.5626 & 26.2247 & 2.8945 \\
& & & & & & \\
$b / a_{B}^{*}$ & $c_{0}$ & $c_{1}$ & $c_{2}$ & $c_{3}$ & $c_{4}$ & $c_{5}$ \\
\hline 0.5 & 0.0133105 & 15.6936 & 29.9494 & 0.499742 & 1.01564 & 0.132745 \\
1.0 & 0.00314 & 13.6338 & 61.2866 & 0.5366 & 0.63904 & 0.07028 \\
\hline \hline
\end{tabular}

$=E\left[\rho^{(1)}\right]+E\left[\rho^{(2)}\right]+E_{I}\left[\rho^{(1)}, \rho^{(2)}\right]$ includes contributions from single wires and an interaction term describing the interwire Coulomb interaction written as

$E_{I}\left[\rho^{(1)}, \rho^{(2)}\right]=\frac{1}{2} \int d z^{\prime} \int d z \frac{\left[\rho^{(1)}(z)-\rho_{0}\right]\left[\rho^{(2)}\left(z^{\prime}\right)-\rho_{0}\right]}{\left[d^{2}+\left(z-z^{\prime}\right)^{2}\right]^{1 / 2}}$,

where $\rho^{(i)}(i=1,2)$ denotes the charge densities in different wires. The above formulation neglects the tunneling effects between the quantum wires, which should be a good approximation for $d / b \gtrsim 1$. Furthermore, the full correlation energy of the double-wire structure is not incorporated. Since the interwire correlation effects are much weaker than the intrawire correlations, ${ }^{25}$ we believe that this approximation is also justified. In Fig. 3 we show the energy difference $\Delta \varepsilon / \lambda^{2}$ between the solid and fluid phases for a double-wire system as a function of $r_{s}$. As in the double-layer electron systems, we observe that the critical density at which the Wigner transition occurs increases as the separation distance $d$ decreases. In particular, for a double-wire system with $b=a_{B}^{*}$ in both wires, we locate the freezing transition at $r_{s c}=5.475, r_{s c}$ $=5.654$, and $r_{s c}=5.711$ for wire separations $d=a_{B}^{*}, 2 a_{B}^{*}$,

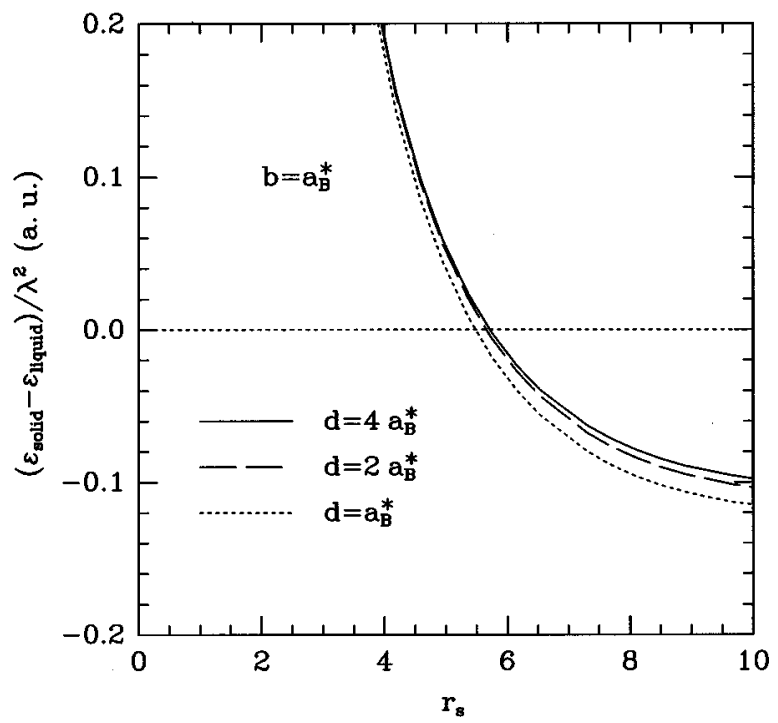

FIG. 3. The difference in the average energy per particle $\left(\varepsilon_{\text {solid }}-\varepsilon_{\text {liquid }}\right) / \lambda^{2}$ (in a.u.) as a function of $r_{s}$ for a double quantum wire system. Radius of the individual wires is $b=a_{B}^{*}$, and the separation distances are $d=a_{B}^{*}$ (dotted line), $2 a_{B}^{*}$ (dashed line), and $4 a_{B}^{*}$. 
and $4 a_{B}^{*}$, respectively. In contrast to the large reduction in $r_{s c}$ with decreasing $d$ in double-layer systems, we find that the crystallization density decreases only slightly by the presence of an extra quantum wire. Nevertheless, it may be more feasible experimentally to observe the crystallization phenomenon in double-wire structures as they are beginning to be fabricated. ${ }^{26}$ Similar considerations as in the case of single-quantum-wire systems, with the additional approximation of $\langle V\rangle=e^{2} /\left(r_{s}^{2} a_{B}^{* 2}+d^{2}\right)^{1 / 2}$ for the average interwire potential, yield the following relation between the critical density parameter $r_{s c}$, the lateral wire width $b$, and the separation distance $d$ :

$$
\Gamma_{0}=\frac{96 r_{s c}^{2}}{\pi^{2}}\left[\frac{1}{\sqrt{r_{s c}^{2}+\left(b / a_{B}^{*}\right)^{2}}}+\frac{1}{2} \frac{1}{\sqrt{r_{s c}^{2}+\left(d / a_{B}^{*}\right)^{2}}}\right],
$$

at freezing, for some constant $\Gamma_{0}$. Such qualitative arguments can also be extended to the cases where quantum wires have different radii and number densities. As for the single-quantum-wire system, the simple estimate predicts crystallization for all $b$ and $d$, for large enough $\Gamma_{0}$.

In summary, we have examined the possibility of Wigner crystallization in single- and double-quantum-wire systems, within a density-functional-theory approach, using the correlation energy given by the self-consistent-field scheme. ${ }^{19} \mathrm{We}$ have found that for experimentally attainable quantum wire widths and electron densities, freezing of a Q1D electron gas should be observable. Although we have used the exchangecorrelation energies calculated for a specific model of a Q1D electron gas, we surmise our results for the Wigner crystallization will qualitatively remain true for other models. An important limitation could be the self-consistent-field approach employed in the calculation of the exchangecorrelation energies. In view of the importance of the correct liquid state input in the density-functional theories of freezing, it would be most useful to have accurate ground-state energies and structure factors for Q1D electron systems in the more accurate QMC and hypernetted-chain-type calculations. Since the disorder effects significantly alter the Wigner crystallization picture, ${ }^{27}$ it would be interesting to investigate similar mechanisms in Q1D structures.

This work was partially supported by the Scientific and Technical Research Council of Turkey (TUBITAK). We thank Dr. C. Bulutay for fruitful discussions.
${ }^{1}$ D. V. Averin and K. K. Likharev, in Mesoscopic Phenomena in Solids, edited by B. Alshuler et al. (Elsevier, Amsterdam, 1991).

${ }^{2}$ For a review see, e.g., R. Cingolani and R. Rinaldi, Nuovo Cimento 16, 1 (1993).

${ }^{3}$ E. Wigner, Phys. Rev. 46, 1002 (1934).

${ }^{4}$ For a collection of recent reviews, see Physics of the Electron Solid, edited by S.-T. Chui (International Press, Cambridge, 1994).

${ }^{5}$ Crystallization or melting of a quantum one-component plasma is also of great interest. See, for instance, M. D. Jones and D. M. Ceperley, Phys. Rev. Lett. 76, 4572 (1996), and references therein.

${ }^{6}$ C. C. Grimes and G. Adams, Phys. Rev. Lett. 42, 795 (1979); F. Gallet, G. Deville, A. Valdes, and F. I. B. Williams, ibid. 49, 212 (1982).

${ }^{7}$ H. J. Schulz, Phys. Rev. Lett. 71, 1864 (1993).

${ }^{8}$ A. Holas and N. H. March, Phys. Lett. A 157, 160 (1991).

${ }^{9}$ D. V. Averin and Yu. V. Nazarov, Phys. Rev. B 47, 9944 (1993).

${ }^{10}$ B. Sutherland, Phys. Rev. Lett. 35, 185 (1975).

${ }^{11}$ A. L. Ivanov and H. Haug, Phys. Rev. Lett. 71, 3182 (1993); Phys. Status Solidi B 188, 125 (1995).

${ }^{12}$ T. V. Ramakrishnan and M. Yussouff, Phys. Rev. B 19, 2775 (1979).

${ }^{13}$ G. Senatore and G. Pastore, Phys. Rev. Lett. 64, 303 (1990).

${ }^{14}$ For reviews and various applications, see G. Senatore and G. Pastore, in High Pressure Equation of State: Theory and Appli- cations, CXIII Varenna School, Varenna, 1989, edited by X. S. Eliezer and R. A. Ricci (North-Holland, Amsterdam, 1991); A. R. Denton, P. Nielaba, and N. W. Ashcroft, J. Phys.: Condens. Matter 9, 4061 (1997).

${ }^{15}$ N. Choudhury and S. K. Ghosh, Phys. Rev. B 51, 2588 (1995).

${ }^{16}$ M. P. Das and J. Mahanty, Phys. Rev. B 38, 5713 (1988).

${ }^{17}$ G. Goldoni and F. M. Peeters, Europhys. Lett. 37, 293 (1997); 38, 319(E) (1997).

${ }^{18}$ A. Gold and A. Ghazali, Phys. Rev. B 41, 7626 (1990).

${ }^{19}$ L. Calmels and A. Gold, Phys. Rev. B 52, 10841 (1995).

${ }^{20}$ C. N. Likos, S. Moroni, and G. Senatore, Phys. Rev. B 55, 8867 (1997).

${ }^{21}$ P. M. Platzman and H. Fukuyama, Phys. Rev. B 10, 3150 (1974).

${ }^{22}$ L. Świerkowski, D. Neilson, and J. Szymański, Phys. Rev. Lett. 67, 240 (1991).

${ }^{23}$ H. C. Manoharan, Y. W. Suen, M. B. Santos, and M. Shayegan, Phys. Rev. Lett. 77, 1813 (1996).

${ }^{24}$ V. I. Valtchinov, G. Kalman, and K. B. Blagoev, Phys. Rev. E 56, 4351 (1997); J. S. Thakur and D. Neilson, Phys. Rev. B 56, 10 297 (1997).

${ }^{25}$ N. Mutluay and B. Tanatar, J. Phys.: Condens. Matter 9, 3033 (1997).

${ }^{26}$ K. Komori, X.-L. Wang, M. Ogura, H. Matsuhata, and H. Imanishi, J. Appl. Phys. 68, 3787 (1996).

${ }^{27}$ S.-T. Chui and B. Tanatar, Phys. Rev. Lett. 74, 458 (1995); J. S. Thakur and D. Neilson, Phys. Rev. B 54, 7674 (1996). 